This is an electronic reprint of the original article. This reprint may differ from the original in pagination and typographic detail.

Author(s): Tyrväinen, Pasi

Title: $\quad$ Estimating Applicability of New Mobile Content Formats to Organizational Use

Year: $\quad 2003$

Version:

Please cite the original version:

Tyrväinen, P. (2003). Estimating Applicability of New Mobile Content Formats to Organizational Use. Proceedings of the 36th Hawaii International. USA: IEEE Computer Society Press. 10.

All material supplied via JYX is protected by copyright and other intellectual property rights, and duplication or sale of all or part of any of the repository collections is not permitted, except that material may be duplicated by you for your research use or educational purposes in electronic or print form. You must obtain permission for any other use. Electronic or print copies may not be offered, whether for sale or otherwise to anyone who is not an authorised user. 


\title{
Estimating Applicability of New Mobile Content Formats to Organizational Use
}

\author{
Pasi Tyrväinen \\ Faculty of Information Technology, University of Jyväskylä, Finland \\ pasi.tyrvainen@jyu.fi
}

\begin{abstract}
Innovations on information and communication technology reshape organizational communication. Our ability to estimate applicability of new technologies and content formats to organizational use has been limited to generic quantitative methods at the level of technologies and industries and elaborate qualitative methods targeting specific phenomena at the organizational level. In this paper we estimate quantitatively the applicability of MMS, SMS, XHTML, and XML to organizational use based on an analysis of all communication of an organization unit. We enumerate over 700 communication genres using a genre-based information systems planning method, categorise them with taxonomy of communication forms, and summarise the results quantitatively per category. SMS, XHTML, and especially XML seem to match the contemporary communication practices of the organization to a much higher extent than MMS. Affect of changes in organizational communication practices and generality of the results are discussed in the end as well as applicability of the method for other purposes.
\end{abstract}

\section{Introduction}

Innovations on information processing equipment and digital communication media have increasingly reshaped organizational communication and the whole concept of organizing during the last few decades $[9,14]$. Consumers have followed business users in adopting mobile telephones, hand-held computers, and PDA equipment to daily use. Borderlines in between PC and telephone equipment are vanishing due to the extensive use of IP protocol in all device categories. Due to this digital convergence we are merely using the term Information and Communication Technology or ICT instead of separating IT and telecom.

New ICT technologies are being introduced frequently, including Multimedia Messaging Service (MMS), XHTML mark-up, and Wireless Application Protocol (WAP) [13]. Product designers are facing problems in choosing technologies for the platforms of their future products as customer behaviour often contradicts their expectations - Short Message Service (SMS) has been extremely successful while Iridium and WAP failed to reach their commercial targets. Market research organizations try to estimate consumer markets of new technologies based on generic functions applicable for generic consumer groups or industries. However, these quantitative estimates are more or less based on technologies used, market segments, or particular product categories rather than the specific requirements set by the organizational communication to the medium and devices used.

In this paper we analyse the potential of using SMS, MMS, XML, and XHTML for encoding content and communication mediated using wireless ICT devices. The analysis is based on a detailed description of contemporary communication forms collected from a typical contemporary organization by using a genre-based method for information systems planning [10]. Each of these communication genres was annotated with metadata describing the volume of communication and the communication media and format. Based on this data, we estimate the amount of communication matching the capabilities of SMS, MMS, XML, and XHTML (These definitions are not strictly speaking formal, "de jure" standards, but are commonly referred to as "standards", also here in short.) Our specific target is to answer the questions: What are the upper limits for number of MMS and SMS messages sent per person per year in a target organization? And based on this, try to give some background for answering the question: How large potential do MMS, SMS, XHTML and XML have in business use?

The paper is organized as follows. Section 2 reviews the underlying theories of organizational communication, the method for genre identification and metadata annotation, the categories of organizational communication, and the metrics applied for quantitative data. Section 3 describes the target organization of the case study, the research process, and reports the resulting data. Section 4 interprets the data, estimates the potential change of communication practices, and discusses some 
aspects of the study. Section 5 concludes and suggests tracks for subsequent research efforts.

\section{Theoretical background}

\subsection{Genres of organizational communication and genre identification}

Yates and Orlikowski [15] defined the concept of a genre of organizational communication as a typified and recurrent communicative action that can be identified by its communicative purpose(s) and, to some extent, by its form(s). A genre can be more or less widely recognized and enacted among people within the community [16]. Examples of communication genres include an order confirmation, weekly sales summary, annual report, and a project meeting. Each organization has a repertoire of genres that can be identified with it at a certain moment of analysis [9]. A set of genres can also interrelate in a wider communicative process thus forming systems of genre [2], called also a genre system [17].

Empirical efforts to identify organizational genre repertoires have reported hundreds of genres that can be found in organizations, denoting that this approach would provide a detailed, yet comprehensive and comprehensible, view on organizational communication $[4,12]$. At least one practical method to identify and analyse genre repertoires has been established and applied to a number of organizational contexts, "A Genre-Based Method for Information Systems Planning" [10]. We adopted and tailored this method for information collection for our purposes. The method variant used for our research purposes incorporates the following basic concepts:

- Information resource of an organization intended to be analysed; this domain is structured by means of the internal PUI entities (see below) and genres identified.

- Stakeholders having interests to participate in the analysis.

- PUI entities producing or using information subsumed in the information resource (PUI = Producer or User of Information), including both external organizations and the internal entities of the organization. Depending on the organization the PUI entities can instantiate as business processes, departments, functions, organization structures, roles, or even individual human beings.

- Genres, as defined above.

- Properties of the identified genres defining the metadata gathered about the genres from the stakeholders for the analysis.
The steps in this tailored method are the following:

1. Identify stakeholders of the analysis.

2. Identify PUI entities.

3. Identify and name genres.

4. Refine the properties / metadata needed.

5. Gather metadata about the genre properties from participants.

6. Analyse the genre-based metadata.

In step 1 the parts of the organization and the persons needed for data collection are identified. Typically, group meetings are conducted to identify PUI entities in step 2. The genres are identified among these PUI entities in step 3 as a named collection of information send in between PUI entities within the organization or in between a PUI entity in the organization and an external PUI entity. In step 4 the properties to be identified for further analysis are refined. The properties needed for our purposes are defined in the following chapters. Also the metadata collection in step 5 and the analysis in step 6 - as tailored for our research purposes - are described in more detail below. For further details of the overall identification process of genre repertoires, see the original method description [10].

\subsection{Categories of organizational communication forms}

Genre theory introduces the concept of genre to structure organizational communication, and declares that certain media can be characteristic for certain genres [15]. In a literary review we found two taxonomies related with our need for classify genres according to their media characteristics. Yoshioka et. al. [18] proposed the use of taxonomic categories on the dimensions for genres and genre systems reflecting the communicative questions why, what, how, when, where, and how $(5 \mathrm{~W} 1 \mathrm{H})$. Out of these, the question "How" addresses the form of a genre/genre system referring to observable features including structural elements, medium, and linguistic features, but does not provide a taxonomy for the features of media matching the needs of this study. In their study Zmud et. al. [19] categorize communication into 14 communication channels within 5 categories: face-to-face, group, written, traditional communication technologies, computer-mediated communication technologies. Although being a step to the right direction, this taxonomy does not provide sufficient level of detail for the analysis of various ICT media.

For analysing the applicability of content standards we constructed a categorization of organizational communication forms that emphasises the ability of computers to interpret and process the information. This taxonomy (see Figure 1) divides the information flows 
first into communication using medium and not, divides communication using medium further to stored and nonstored, divides the stored information further into digital and non-digital information, and further elaborates the digital communication forms in according to the increasing ability of computers to interpret the procedural semantics of the encoding and structure of the contents. For a more detailed description of the categories of communication forms, see Table 1 in Appendix 1.

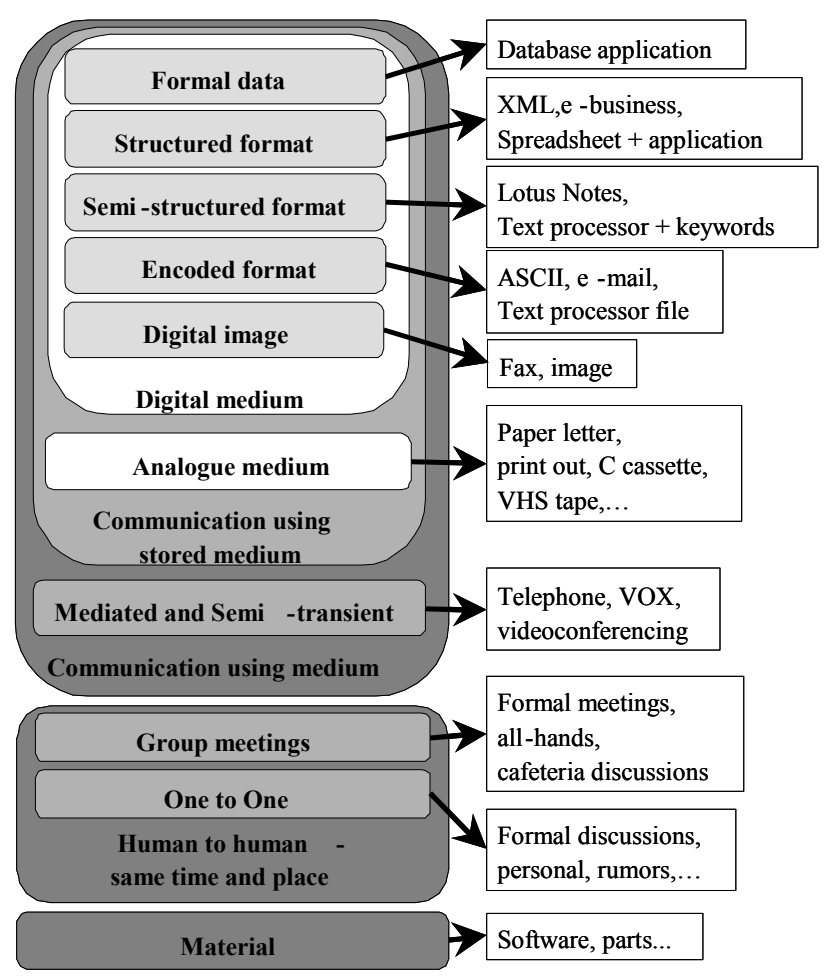

Figure 1. Taxonomy of organizational communication forms used in this study.

For our purposes, this taxonomy enables us to match the characteristics of the analysed standards with the communication genres categorized simply by categorising the standards or technologies in question. In our case the categories are as follows:

- Multimedia Messaging Service matches the characteristics of category Digital image, i.e. transmission of plain bitmaps or pictures encoded for picture transmission.

- Short Message Service matches the characteristics of category Encoded in transmitting textual messages with character encoding.

- XHTML matches best the characteristics of category Semi-structured as the structure tagged in XHTML messages or pages can be utilized to some extent by the applications, although not to the extent of XML.

- $\quad$ XML enables applications to interpret the semantics of the data to a much higher extent than XHTML, thus being categorised to class Structured.

\subsection{Metrics and calculation process}

The inputs of the calculation are the properties gathered from each communication genre during the metadata gathering phase. These were:

- Category or categories of communication forms used by the genre.

- Number of unique instances (UI) per time. Unique instances refer to the distinct instances of a genre communicated excluding duplicates, e.g. a single mail message sent to several recipients or a broadcast is considered to be a single unique instance. A single annual report is being produced per year, whereas several invoices can be produced daily.

- Copies or instances per UI, which refer here to the average number of copies delivered to distinct receivers of the UI. A project status meeting is the only copy of the communication act while a corresponding digital document - the memorandum - can be copied by e-mail and sent to tens of persons.

- Size of instances. The average amount of information per instance of a genre. This varies from multi-volume contracts and manuals to acknowledgement notes or "Please call ..." notes.

The size of UI (equal to size of instance) is measured in Pages that refer to amount of information equal to a view of the size of a visual letter / A4 page. For the stored forms of communication this matches the number of printed pages in a paper version of a typical instance of a genre. A Page is considered to be roughly equivalent to 13 kilobytes of plain ASCII encoded text or about a megabyte of bits of a digital image. This approach aims at filtering out the physical size, size in bytes, or other medium-dependent aspects as technical details - moving over from ASCII text to bitmap increases the amount of bits by factor of 100 or 1000 but does not affect the amount of information communicated nor the size of instances measured in Pages. For further discussion on metrics on information see [5, 6, and 7].

The calculation process manages the metadata of communication genres in a detailed level and in summary levels in three orthogonal dimensions. The dimensions are: 
- Genres, i.e. calculations per one genre or summaries of all genres.

- Communication forms, i.e. calculations per one category of communication forms, per a group of categories of communication forms (such as Stored or Digital communication forms only), or per all communication forms.

- Measurement units are further composed out of 3 dimensions:

- UI or Copies, i.e. are all copies of genre instances calculated.

- Instances or Pages, i.e. counting items or measuring volumes.

- Absolute or proportional, i.e. absolute or percentage values.

Figure 2 represents the data elements of the calculation process. The calculation process takes the metadata collected per genre (rows in Figure 2) as its inputs. For each element - i.e. for each combination of a genre and a category - the process calculates four values. They are the four absolute measurement units, "Annual Number of UI" and "Annual Number of Copies" as well as "Annual UI Volume" and "Annual Volume" measured in Pages. The results are summed up per each category of communication forms, i.e. per columns in Figure 2. This produces four absolute measurement values for each category. Further, these values are summed up for groups of categories of communication forms, e.g. to four absolute measures of communication using Stored medium, four for Digital medium, as well as four values summing up all communication of the organization. These are referred to with names like (number of estimated) "Organizational Total Annual UI" or "Organizational Total Annual Volume" (in Pages).

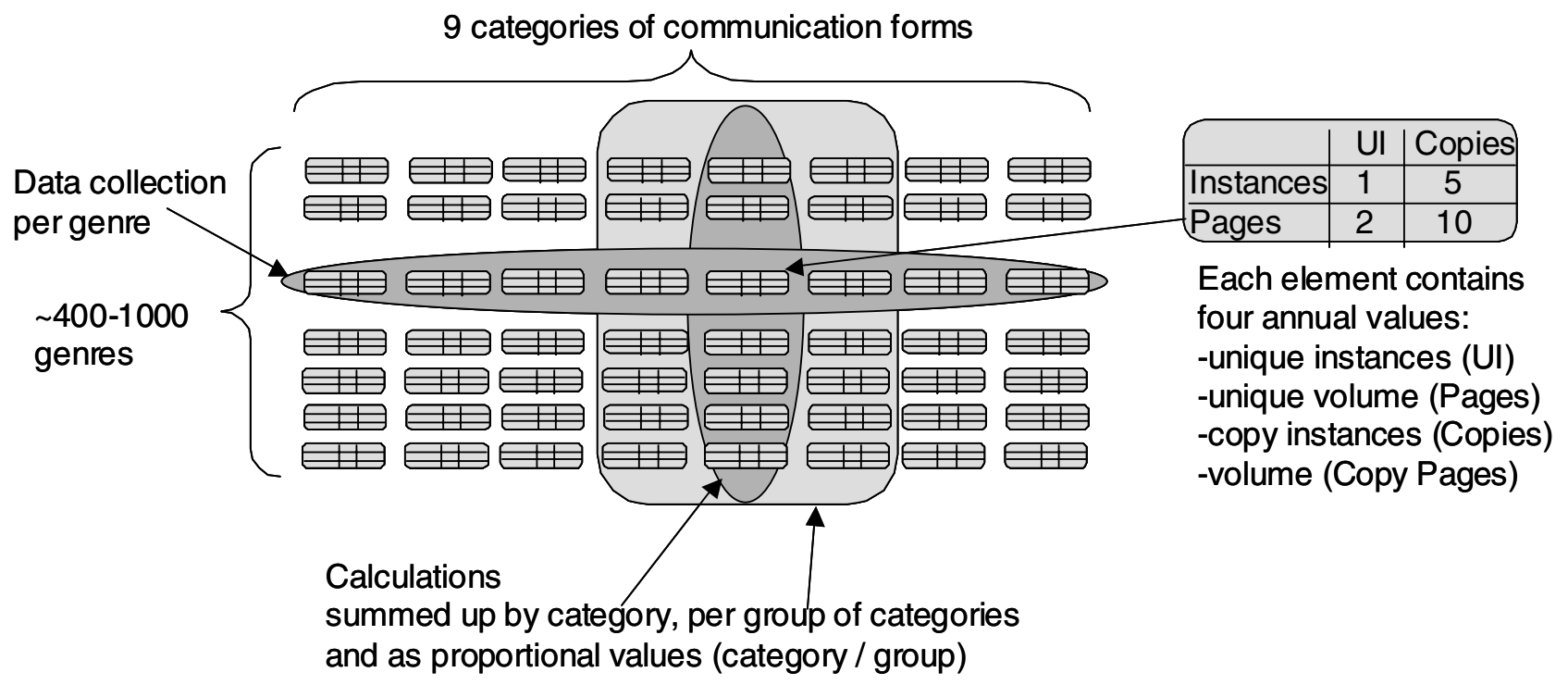

Figure 2. Data elements of the calculation process.

The proportional values are formed by comparing absolute values of a category(-ies) of communication forms with absolute values of a group of categories of communication forms. For example, "Organizational Stored UI Volume Percentage of Semi-structured communication forms" is the percentage of "Organizations Annual UI Volume of Semi-structured communication forms" divided by "Organizations Annual UI Volume of Stored communication forms". As the applicability of the measurement unit "Page" is somewhat contradictory with the communication forms that do not use tangible medium, we limit the use of the volume measures to the forms using medium, referred hereafter with names like "Organizational Medium UI Volume...", e.g. "Organizational Medium UI Volume Percentage of Digital communication forms". Using these definitions we are also able to give a precise definition for "Degree of digitalisation" of organizational communication, as "Organizational Stored Volume Percentage of Digital communication forms" or as "Organizational Medium Volume Percentage of Digital communication forms". 


\section{A case study}

\subsection{The target organization and the research process}

The target organization was an independent unit of a multinational high-tech corporation, involving ca 400 employees, located in Finland. The identification process of the genre repertoire was performed in fall 1999. It included 8 sessions with 3-5 participants, lasting 3 hours each, with a total of 3,5 person weeks of effort from the target organization and 8 person weeks from the researchers, including the problem analysis. A total of 744 communicative genres were identified and named.

Two persons of the target organization, P1 and P2, participated in the metadata collection of the genres for our research purposes during the summer 2000. Both of them have several years of experience with core processes, process development, and information systems of the organization. P1 can be best characterized as a manager of a central process while P2 carries the overall responsibility for the information systems of the target organization and has somewhat more overall knowledge over the hundreds of genres in the organization. The effort spent on the analysis of genres totalled five person days from the two (extremely busy) persons, $\mathrm{P} 1$ and $\mathrm{P} 2$.

Out of the over 700 genres originally identified, a subset of $10 \%$ was analysed both by P1 and P2 in order to save time and effort. These were chosen by picking up every 10th genre from the full list of genres - ordered in the order of identification - to ensure that the subset will represent well the full set. This subset is referred to as the "Subset" in later diagrams. As persons P1 and P2 were not familiar with all the genres, the unknown ones were filtered out from of both of the data sets. Also the genres categorized as "Material" were filtered out. The resulting "Full" filtered data set included 598 genres and the filtered subset included 54 genres. Some examples of the genres and metadata collected are presented in Table 2 in Appendix 1.

\subsection{Results}

Figure 3 represents the distribution of categories of communication forms used in the target organization at the level of genres. The three series represent the values for the Full data set as annotated by person P1 and the Subset as annotated by $\mathrm{P} 1$ and $\mathrm{P} 2$. The percentages in series "P1 Full" show that most genres $(62,7 \%)$ were communicated with some Encoded form of communication; word processing documents, mail messages etc. Almost third of the genres $(31,9 \%)$ were communicated using paper or other Analogue media. On an average, a genre used communication forms from 1,77 categories, being rather soft (about softness of genres, see [11]).

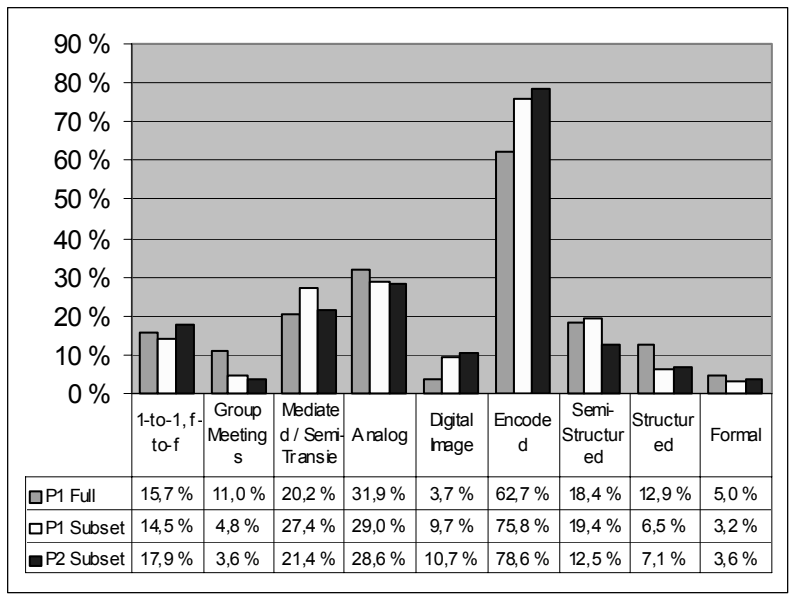

Figure 3. Distribution of communication forms used by the genres of the study.

The number of annual unique instances for the subset of 54 genres was calculated based on the measures. This resulted in about 49'000 annual unique instances for the 54 genres implying organization's total annual UI to be about $680^{\prime} 000$. Table 3 presents the figures calculated for the subset of 54 genres as well as the figures extrapolated to the organization's total 744 genres identified.

Table 3. Organization's total Annual Volumes

\begin{tabular}{|l|l|l|}
\hline & $\begin{array}{l}\text { Subset } \\
\text { (54 genres) }\end{array}$ & $\begin{array}{l}\text { Estimated } \\
\text { organization' } \\
\text { s total }\end{array}$ \\
\hline Annual Unique Instances & $49^{\prime} 000$ & $680^{\prime} 000$ \\
\hline Annual UI Volume / Pages & $103^{\prime} 000$ & $1^{\prime} 400^{\prime} 000$ \\
\hline Annual Copies & $170^{\prime} 000$ & $2^{\prime} 300^{\prime} 000$ \\
\hline Annual Volume / Pages & $590^{\prime} 000$ & $8^{\prime} 100^{\prime} 000$ \\
\hline
\end{tabular}

Figure 4 represents the distribution of Organization's Annual UI, Copies, UI Volume, and Volume per the categories for the Subset in proportional values. From this we can see that most instances of communication used Structured communication forms (64\% of UI and $54 \%$ of Copies) while paper documents seem to dominate communication when measured by Volume of copies (41\%). The Encoded communication forms were dominating in the number of genres (see Figure 3), but they seem to generate less instances per genre when compared with the previous categories. 


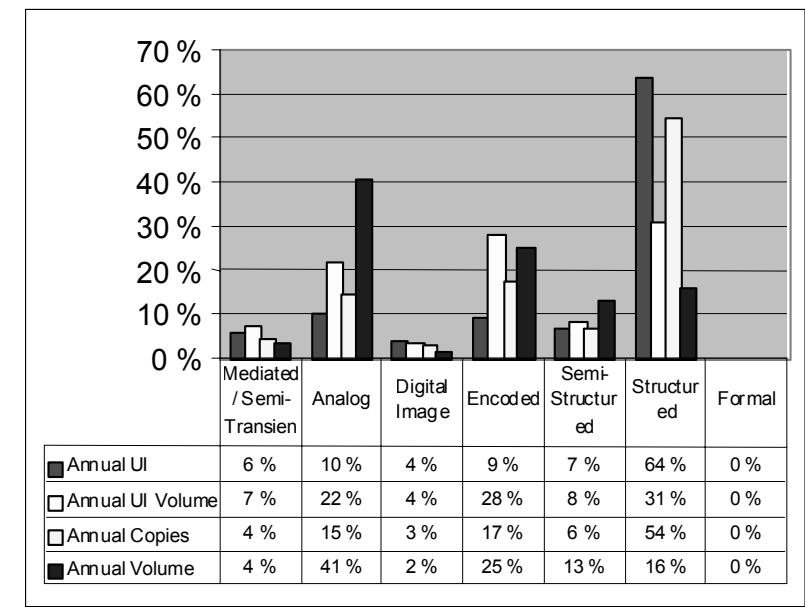

Figure 4. Proportions of categories of communication forms in the Subset

From the viewpoint of analysing adoptability, our aim was to quantify the communication applicable for the content formats listed. This was done by dividing the estimates of organization's total Annual Volumes for the four absolute measures (in Table 3) by the number of employees in the organization. Table 4 lists these four values (on the right) for each Mediated category of communication forms (column "CCF") as well as positions the standard-like formats to the closest category based on the properties of the formats (second column from the left). I.e. the potential of use for each of the standards in the target organization can be read in form of annual figures per person.

Table 4 Annual communication per an employee.

\begin{tabular}{|l|l|r|r|r|r|}
\hline \multicolumn{1}{|c|}{ CCF } & Standard & \multicolumn{1}{c|}{ UI } & $\begin{array}{c}\text { UI } \\
\text { Volume }\end{array}$ & Copies & $\begin{array}{c}\text { Copy } \\
\text { Volume }\end{array}$ \\
\hline Formal & & 0 & 2 & 1 & 6 \\
\hline Structured & XML & 1080 & 1080 & 3100 & 3200 \\
\hline $\begin{array}{l}\text { Semi- } \\
\text { Structured }\end{array}$ & XHTML & 115 & 300 & 370 & 2700 \\
\hline Encoded & SMS & 160 & 1000 & 1000 & 5050 \\
\hline $\begin{array}{l}\text { Digital } \\
\text { Image }\end{array}$ & MMS & 65 & 125 & 160 & 350 \\
\hline Analogue & & 175 & 760 & 840 & 8200 \\
\hline $\begin{array}{l}\text { Mediated/ } \\
\text { Semi- } \\
\text { Transient }\end{array}$ & GSM & 100 & 250 & 260 & 720 \\
\hline $\begin{array}{l}\text { TOTAL } \\
\text { Mediated }\end{array}$ & & 1700 & 3500 & 5700 & 20200 \\
\hline
\end{tabular}

In addition to the applicable category of communication forms we are interested in other aspects of communication relevant for adoption of the standards. E.g., the SMS short message service has an upper limit of the size of the data sent. How large portion of the communication of the Encoded category could be sent with one SMS vs. has to be cut into several messages? Also the first equipment making use of MMS may be limited into small message sizes due to practical limitations, e.g. to individual still images. Figure 5 represents the distribution of instance size measured in Pages for all the genres in the Subset.

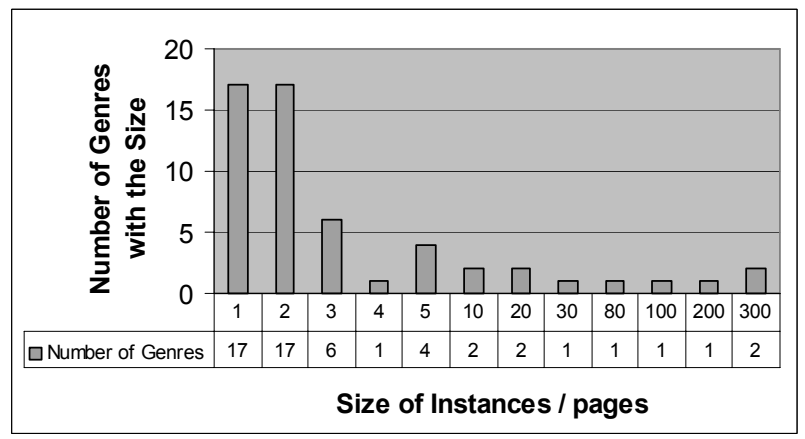

Figure 5. Size of instances in the Subset

To focus on the sizes of messages applicable for SMS and MMS we also calculated the UI and Copies for Encoded and Digital forms of communication. Out of the annual 4'600 unique messages and 30'000 copies in Encoded format in the Subset, $36 \%$ and $38 \%$ were classified to transmit a single page of information, respectively. These small ones were instances of such genres as "Change in schedule", "Permission to submit a tender", and "Packaging list". For the 1'900 and 4'700 unique messages and copies in Digital image formats only $26 \%$ and $11 \%$ were classified to transmit a single page while the others included more content. For both of these categories, $90 \%$ of the instances and UIs were 3 Pages or smaller.

In addition to the size of the messages and the category of communication form the communication in the target organization can also be analysed against other aspects relevant for adoption of mobile communication formats, e.g. processing requirements set by XML encoding, processing time, latencies, memory consumption, and security considerations - as long as relevant metadata about the communication genres has been collected during the process along with the CCF and quantitative metadata presented in this method (for further, see [10]). However, detailed analysis on these other dimensions as well as interviews on communication practices and other qualitative data collection was considered to be out of the scope of this work. 


\section{Result analysis}

\subsection{Contemporary communication forms}

Assuming that the employees of the target organization would maximise the use of mobile equipment in their daily tasks, how much information could be transmitted using the mobile communication formats without other changes in their communication practices, i.e. assuming that the communication forms would remain the same?

According to Table 4 each employee sends and receives annually total 1000 communication instances using Encoded formats, out of which 160 are unique (i.e. on an average, 7 copies of a mail message or office document are distributed). If we count only the ones with size of a single Page (38\%) this means that the potential for SMS would be annually 380 messages per person including copies or 57 unique messages.

Assuming the limit of a Page to be valid also for MMS we end up with the figure of 17 messages of the Digital image communication forms to be applicable for MMS per employee in a year. This is less than a third of the potential for unique SMS messages or less than $5 \%$ of the potential for the copies of SMS messages. Note, however, that the contemporary Digital image communication forms in the organization in question include only the use of fax, while the Encoded communication forms include commonly used e-mail and word processor files, that will be used also for one-page messages instead of SMS, whenever the persons communicating are at their premises or connected to the network. Note also that neither the use of digital cameras nor WLAN were captured by the genre analysis although they were already adopted by the target organization at the times of data collection of this study.

The features of XHTML match best the Semi-structured communication forms of the categorisation, although this classification is less self-evident as with the other formats. In number of Annual Unique Instances per employee (Table 4) the figure of this category is close to the figure of Encoded communication forms (115 vs. 160), but the figures for UI Volume and in number of Copies are only one third of the respective figures of Encoded forms. However, two thirds of Encoded communication instances transmit more than one Page of information, which communication matches better with the capabilities of XHTML rather than the ones of SMS. This implies that equipment with large displays and capability to handle up to 3 pages of information either in XHTML format or by combining several SMS messages would match majority of the communication using Encoded (and Semistructured) communication forms in this organization while equipment capable to handle single SMS messages are much less useful.
In the target organization, the communication using Structured forms of communication was increasing rapidly and extensively in volume (see Table 4 and Figure 4). Most of this managed small chunks of information communicating single Page messages in between applications (e.g. applications using spread-sheet programs as user interfaces). Along the adoption of ebusiness applications this category of communication has increasingly been adopted to organizations in the form of e-business message definitions, such as RosettaNet and ebXML, which are based on the XML definition.

\subsection{Potential changes in communication forms}

So far the result analysis has been based on the contemporary communication practices of the organization, i.e. analysed the media and formats used for each communication genre of the organization at present. Still in year 2000 an average employee sent or received total 8'200 pages of paper information $(42 \%$ all Stored communication forms), including copies of large manuals, tenders etc. It seems very unlikely that a major portion of these would be communicated using MMS or SMS, rather than using Structured or Semi-structured communication forms. With the adoption of e-business formats the use of Structured communication forms has been increasing rapidly and is likely to gain even more momentum in the near future from the genres now using Encoded communication forms.

Based on this study the consumer market seems to be more promising for MMS if only contemporary communication practices are observed. However, in the business environment the capability of MMS "rich messages" to include both pictures and textual information may make it popular for some genres that are now using Encoded communication forms, such as genres related with marketing and personnel management. In addition, some of the non-mediated and Mediated / Semi-Transient communication forms may adopt special MMS equipment, e.g. to enable mediated face-to-face discussions while travelling replacing specialized videoconference equipment to some extent. However, these changes depend much on the availability of suitable technical equipment and the critical mass [8] needed for general technology adoption.

\subsection{Discussion}

In this research we have made use of the genre-based analysis method in dividing the communication of the target organization into a level of detail sufficient for qualitative and quantitative analysis. The data was collected bottom up using the processes of the 
organization as the baseline for the analysis. We ended up with results (Table 4) such that each employee sent and received on an average 5'700 communication instances per year using some mediated forms of communication, i.e., 26 instances and 92 Pages per day. Are these figures realistic?

Landauer [5] estimated that people take in and remember about a byte in a second, i.e. up to 675 KBytes or about 300 Pages during office hours. Thus about 90 Pages per day seems to be realistic as it excludes verbal communication and not all of the 90 Pages are read or written rather than just skimmed and forwarded. Further, this figure includes all the communication related with the business of the organization, but excludes private use of communication equipment, that will add in to the total amount of communication (see a recent study on use of mobile services [1]).

In the project "How Much Information?" at Berkeley Lyman et. al. [7, Executive summary, table 6, p. 6] estimated about 7'500 million titles of unique office documents to be produced worldwide annually, adding up to 19 Terabytes - or 7'500 million pages, each containing 2,5 KBytes of plain text per page [7, Print, table 1, p. 2], i.e. one page per title. These accounted for $81 \%$ of all the printed material produced in the world. In our study, this matches with the categories of Analogue and Encoded communication forms - the category of Encoded communication forms includes both word processor files transmitted via e-mail and e-mail used as such. In the target organization, each person sent and received annually a total of $335(160+175)$ unique Encoded and Analogue communication instances and 1760 Pages. Assuming half of them to be sent and half received and further, $81 \%$ of the sent ones to be produced using word processors, the number of employees needed to produce the annual 7'500 million unique documents and Pages would be 55 millions and 10,5 millions, respectively.

\section{Summary, conclusions, and further research}

This research used communicative genres as the conceptual means to structure organizational communication for data collection. The applicability of SMS, MMS, XHTML, and XML were analysed by classifying the organizational communication according to taxonomy of communication forms and matching the standards against these categories. In addition, the limited size of SMS and MMS messages was taken into account. The results were quantified using four absolute and proportional measures for the number of unique instances and copies as well as for unique and non-unique communication volumes.
The contemporary (year 2000) communication practices of the target organization include little communication that would match the capabilities of MMS. Thus the use of MMS will require changes in communication practices in addition to adoption of the technology. This likely appearance of new communication genres and ad hoc communication that will take place in the near future due to new MMS technology will be a fruitful target for a case study on genre ecologies as introduced by Ericson [3].

According to the contemporary practices SMS can be applied to a wide number of genres using Encoded communication forms. However, only a third of this communication fit into a single page, while $90 \%$ of these would fit into messages of 3 pages or less in the target organization. This means that the potential for SMS is about 380 annual messages per employee while the rest will match better with other formats, such as XHTML. Thus the total potential for XHTML is round 1000 annual messages per employee (including both Encoded and Semi-structured communication forms) and about 3100 for XML. There exists still a potential of over a thousand annual messages per employee in Analogue and Mediated / Semi-Transient communication forms, mostly on paper, that will add on these figures. Especially the adoption of e-business applications will increase the use of XML as the main Structured communication form. Also some portion of the verbal communication is likely to move over to some of these digital communication forms.

Although these figures represent the status of the whole target organization unit, they still represent only a single organization. However, they are consistent with our preliminary results from two other organizations e.g. in presenting little need for MMS in their contemporary communication practices. But prior to making any generalisations we need to collect and analyse material from several organizations to verify these results. Thus we encourage use of the method for studies in other contexts.

The approach presented here can easily be adopted for estimating applicability of other technologies as well, not only for mobile communication formats. Further, extensions of the metrics for other quantitative aspects of organizational communication need to be tested, e.g.:

- for measuring the impact of technology adoption to the measurable values - does the adoption of an ERP system increase the average number of Annual Unique Instances processed per person? Or increase the percentage of Organizations Annual Digital UI Volume out of Organizations Annual Stored UI Volume, as expected?

- for correlating the impact of the measured values to organizational performance - e.g. to measure impact of digitalisation to business performance of organizations - does it pay off to digitalize $100 \%$ of communication or is $80 \%$ the optimum? 


\section{References}

[1] A. Aarnio, A. Enkenberg, J. Heikkilä, and S. Hirvola, "Adoption and Use of Mobile Services - Empirical Evidence from a Finnish Survey", Proceedings of the 35th Annual Hawaii International Conference on System Sciences, IEEE Computer Society Press 2002.

[2] C. Bazerman, "Systems of Genres and the Enactment of Social Intentions", in Freedman, A. and Medway, P. (ed.) Genre and the New Rhetoric: London, Taylor \& Francis, 1994, pp. 79101.

[3] T. Ericson, "Making Sense of Computer-Mediated Communication (CMC): Conversations as genres, CMC Systems as Genre Ecologies". Proceedings of the 33rd Hawaii International Conference on System Sciences. IEEE Computer Society Press, Los Alamitos California, 2000. CD-ROM.

[4] A. Karjalainen, T. Päivärinta, P. Tyrväinen, and J. Rajala, "Genre-Based Metadata for Enterprise Document Management", in Sprague, R.H., Jr. (ed.) Proceedings of the 33rd Annual Hawaii International Conference on System Sciences; Genre in Digital Documents: Los Alamitos CA, IEEE Computer Society, 2000, pp. CD-ROM.

[5] T. K. Landauer, "How much do people remember? Some estimates on the quantity of learned information in long-term memory", Cognitive Science, 10 (4) Oct-Dec 1986. pp. 477-493.

[6] M. Lesk, "How much information is there in the world?" Technical report, lesk.com, available at http://www.lesk.com/mlesk/ksg97/ksg.html 1997.

[7] P. Lyman, H.R. Varian, J. Dunn, A. Strygin, and K. Swearingen, "How Much Information?" School of Information Management and Systems at University of California at Berkeley, PDF report available from http://www.sims.berkeley.edu/how-much-info/summary.html and related pages. 10.11. 2000 .

[8] M.L. Markus, "Toward a 'Critical Mass' Theory of Interactive Media", in Fulk, J. and Steinfield, C. (ed.) Organizations and Communication Technology: Newbury Park, Sage, 1990, pp. 194-218.

[9] W.J. Orlikowski and J. Yates, "Genre repertoire: The structuring of Communicative Practices in Organizations", Administrative Science Quarterly, 39, 4 (1994), pp. 541-574.
[10] T. Päivärinta, V. Halttunen, and P. Tyrväinen, "A GenreBased Method for Information Systems Planning", in Rossi, M. and Siau, K. (ed.) Information Modelling in the New Millennium: Hershey PA, Idea Group, 2001, pp. 70-93.

[11] U. Schultze, and R. Boland Jr., "Hard and Soft Information Genres: An Analysis of two Notes Databases", in Proceedings of the 30th Hawaii International Conference on System Sciences. IEEE Computer Society Press, Los Alamitos, California, 1997. URL: http://www.mngt.waikato.ac.nz/ejrot/

[12] P. Tyrväinen and T. Päivärinta, "On Rethinking Organizational Document Genres for Electronic Document Management", in Sprague, R.H., Jr. (ed.) Proceedings of the 32nd Annual Hawaii International Conference on System Sciences, IEEE Computer Society Press, Los Alamitos CA, 1999. CD-ROM.

[13] Wireless Application Protocol WAP 2.0 Technical White Paper, WAP Forum, http://www.wapforum.org January 2002. $13 \mathrm{p}$.

[14] J. Yates, Control through Communication: The Rise of System in American Management, Johns Hopkins University Press, Baltimore, 1989.

[15] J. Yates, and W.J. Orlikowski, "Genres of Organizational Communication: A Structurational Approach to Studying Communication and Media", Academy of Management Review, 17, 2., 1992, pp. 299-326.

[16] J. Yates, W.J. Orlikowski, and K. Okamura, "Explicit and Implicit Structuring of Genres in Electronic Communication: Reinforcement and Change of Social Interaction", Organization Science, 10, 1, 1999, pp. 83-103.

[17] J. Yates, W.J. Orlikowski, and J. Rennecker, "Collaborative Genres for Collaboration: Genre Systems in Digital Media", in Sprague, R.H., Jr. (ed.) Proceedings of the 30th Annual Hawaii International Conference on System Sciences: Los Alamitos CA, IEEE Computer Society Press, 1997, pp. 50-59.

[18] T. Yoshioka, G. Herman, J. Yates, and W. Orlikowski, "Genre taxonomy: A knowledge repository of communicative actions", ACM Transactions on Information Systems, 19, 4, 2001, pp. 431-456.

[19] R.W. Zmud, M.R. Lind, and F.W. Young, "An Attribute Space for Organizational Communication Channels", Information Systems Research, 1, 4, 1990, 440-457. 
Appendix 1: Table 1. The formats analysed and the categories of communication forms.

\begin{tabular}{|c|c|c|}
\hline Format & $\mathbf{C C F}$ & Description of the CCF - Category of Communication Forms \\
\hline & Material & $\begin{array}{l}\text { Information objects exchanged in between organizational entities, that cannot be classified as information } \\
\text { exchange in between humans, although were identified as "information objects" important for the } \\
\text { organization. F. ex., software distribution media,, products and parts. }\end{array}$ \\
\hline & \begin{tabular}{|l|} 
1-to-1, \\
f-to-f
\end{tabular} & $\begin{array}{l}\text { Personal face-to-face meetings in the same place and time include both formal person-to-person meetings } \\
\text { and informal, casual meetings, such as discussions in cafeteria, etc. }\end{array}$ \\
\hline & $\begin{array}{l}\text { Group } \\
\text { Meetings }\end{array}$ & $\begin{array}{l}\text { Formal group meetings and informal, spontaneous face-to-face meetings. Memorandums composed } \\
\text { /distributed are categorized to other categories. }\end{array}$ \\
\hline & $\begin{array}{l}\text { Mediated / } \\
\text { Semi- } \\
\text { Transient }\end{array}$ & $\begin{array}{l}\text { Communication used for emulating face-to-face communication with no physical interaction. Mediated data } \\
\text { is transmitted by computers or by other ICT equipment. Examples include phone calls, teleconference and } \\
\text { videoconference. Mediated communication captured and stored temporarily by the machines is called here } \\
\text { semi-transient communication. Typical examples include answering machine messages and VOX. }\end{array}$ \\
\hline GSM & Analogue & $\begin{array}{l}\text { This category includes data stored on some analogue medium, typically paper, microfilm, or VRC/audio } \\
\text { tapes. Mechanical machines can copy this kind of data, but storage and distribution requires often } \\
\text { manipulation and transportation of the physical media. Data management is typically based on metadata } \\
\text { (reference) information stored separate from the data. }\end{array}$ \\
\hline MMS & $\begin{array}{l}\text { Digital } \\
\text { Image }\end{array}$ & $\begin{array}{l}\text { Digital data with no or minimal encoding of semantic information. These formats can be stored, copied and } \\
\text { transmitted by computers, but operations making use of the meaning of the contents or content of the } \\
\text { information object are not available. Typical formats include bitmap images transmitted by fax or scanned } \\
\text { from paper or used for storing digitised documents and graphical encoding formats (CGM, GIFF, JPEG). }\end{array}$ \\
\hline SMS & Encoded & $\begin{array}{l}\text { Digital data intended for human use with encoding applicable for simple generic tools, e.g. searching strings } \\
\text { from an ASCII or UNICODE coded documents or word processing files. Typical formats and tools include } \\
\text { mail messages, word processor files etc. }\end{array}$ \\
\hline XHTML & $\begin{array}{l}\text { Semi- } \\
\text { Structured }\end{array}$ & $\begin{array}{l}\text { Digital data containing both large bodies of natural language interpreted only by humans and structured data } \\
\text { processed both by humans and computers. The amount of structured data is considered sufficient if it is used } \\
\text { by major computer applications, e.g. metadata used for workflow control and sophisticated data manipulation } \\
\text { and retrieval. Examples: Lotus Notes databases with applications, spreadsheet applications, document } \\
\text { templates with applications assuming procedural interpretation for the fields. }\end{array}$ \\
\hline \multirow[t]{2}{*}{ XML } & Structured & $\begin{array}{l}\text { Digital data presented through displays or forms for humans. The whole content of the genre is interpreted } \\
\text { both by humans and computer applications without human interpretation or intervention. Typical tools } \\
\text { include eBusiness messages and XML documents with semantic element definitions or pre-defined } \\
\text { spreadsheet sheets used for collecting data for computerised applications. Examples: collecting hour reports } \\
\text { or customer orders, processed further by administrative, order-processing, or RosettaNet applications. }\end{array}$ \\
\hline & Formal & $\begin{array}{l}\text { Digital data intended for computer use and interpreted by computers. The context needed for interpretation is } \\
\text { defined by a database schema or similar means. No common sense or world knowledge of humans is needed } \\
\text { for this process. }\end{array}$ \\
\hline
\end{tabular}

Table 2. Examples of quantitative metadata of genres collected in the case study.

\begin{tabular}{|c|c|c|c|c|c|c|}
\hline Producer & Genre & User & Categories of communication forms & Annual UI & Copies & $\begin{array}{l}\text { Size in } \\
\text { Pages }\end{array}$ \\
\hline $\begin{array}{l}\text { Product } \\
\text { management }\end{array}$ & $\begin{array}{l}\text { List of chosen } \\
\text { equipment }\end{array}$ & Spare parts & Encoded & tens & some & 2 \\
\hline Sales & Request for support & $\begin{array}{l}\text { Project } \\
\text { organization }\end{array}$ & $\begin{array}{l}\text { Personal, Mediated / Semi-Transient, } \\
\text { Encoded }\end{array}$ & hundreds & some & 2 \\
\hline Dept 1 & Internal training & Dept 2 & Group meeting & some & 1 & 100 \\
\hline Purchasing & Order for work & Contractors & $\begin{array}{l}\text { Mediated, Digital image, Encoded, } \\
\text { Structured }(60 \%)\end{array}$ & thousands & 1 & 1 \\
\hline Customer & Order & Sales & $\begin{array}{l}\text { Analogue (5/7), Digital image, } \\
\text { Encoded }\end{array}$ & thousands & 1 & 1 \\
\hline Project dept & Schedule changes & Project dept & $\begin{array}{l}\text { One-to-one, Group meeting, Encoded } \\
\text { (dominating) }\end{array}$ & hundreds & tens & 1 \\
\hline Sales & Permission to tender & Sales & Encoded & hundreds & 1 & 1 \\
\hline Project dept & Project plan & Sales & Encoded & hundreds & some & 3 \\
\hline$\ldots$ & & & & & & \\
\hline
\end{tabular}

